\title{
Uncertainty Principles for Kac Algebras
}

\author{
Zhengwei Liu \\ Department of Mathematics and Department of Physics \\ Harvard University \\ zhengwei.liu@fas.harvard.edu \\ Jinsong $\mathrm{Wu}$ \\ School of Mathematical Sciences \\ University of Science and Technology of China \\ wjsl@ustc.edu.cn
}

June 2, 2016

\begin{abstract}
In this paper, we introduce the notation of bi-shift of biprojections in subfactor theory to unimodular Kac algebras. We characterize the minimizers of Hirschman-Beckner uncertainty principle and Donoho-Stark uncertainty principle for unimodular Kac algebras with biprojections and prove Hardy's uncertainty principle in terms of minimizers.
\end{abstract}

\section{Introduction}

Uncertainty principles for locally compact abelian groups were studied by Hardy [15, Hirschman [16], Beckner [2], Donoho and Stark [9], Smith 23, Tao 24] etc. In 2008, Alagic and Russell [1] proved Donoho-Stark uncertainty principle for compact groups. In 2004, Özaydm and Przebinda [21] characterized the minimizers of Hirschman-Beckner uncertainty principle and Donoho-Stark uncertainty principle for locally compact abelian groups.

Kac algebras were introduced independently by L.I Vainerman and G.I. Kac [27, 28, 29] and by Enock and Nest 10, 11, 12, which generalized locally compact groups and their duals. Furthermore, J. Kustermans and S. Vaes introduced locally compact quantum groups [18. Recently Crann and Kalantar proved Hirschman-Beckner uncertainty principle and Donoho-Stark uncertainty principle for unimodular locally compact quantum groups [7].

Subfactor theory also provides a natural framework to study quantum symmetry. The group symmetry is captured by the subfactor arisen from the group crossed product construction. Ocneanu first pointed out the one-to-one correspondence between finite dimensional Kac algebras and finiteindex, depth-two, irreducible subfactors. This correspondence was proved by W. Szymanski 22. Enock and Nest generalized the correspondence to infinite dimensional compact (or discrete) type Kac algebras and infinite-index, depth-two, irreducible subfactors [14. In general, a subfactor provides a pair of non-commutative spaces dual to each other and a Fourier transform $\mathcal{F}$ between them. It appears to be natural to study Fourier analysis for subfactors. 
In 17, C. Jiang and the authors study uncertainty principles for finite index subfactors in terms of planar algebras. We proved Hirschman-Beckner uncertainty principle and Donoho-Stark uncertainty principle for finite index subfactors. Furthermore, we introduced bi-shifts of biprojections 1 , and use them to characterize the minimizers of the two uncertainty principles.

Moreover, we formalized Hardy's uncertainty principle using the minimizers of the HirschmanBeckner uncertainty principle, and proved it for finite index subfactors. The case for finite-index, depth-two, irreducible subfactors covers the results for finite dimensional Kac algebras. The quantum group community wondered whether the methods in [17] work for infinite-dimensional cases. That is the motivation of this paper.

In this paper, we introduce notions in subfactor theory to unimodular Kac algebras, such as biprojections, bi-shifts of biprojections. For example, the identity of a compact type locally compact quantum group is a biprojection. The Fourier transform transform of a biprojection is a biprojection. We characterize the minimizers the Hirschman-Beckner uncertainty principle and the Donoho-Stark uncertainty principle for unimodular Kac algebras containing biprojections. Furthermore, we prove the Hardy uncertainty principle for such Kac algebras. Our proofs utilize the ideas in subfactor theory [17] and the methods for locally compact quantum groups [18.

Main Theorem 1 (Proposition 3.5. Theorem 3.14). Let $\mathbb{G}$ be a unimodular Kac algebra. For any nonzero $w$ in $L^{1}(\mathbb{G}) \cap L^{2}(\mathbb{G})$, the following statements are equivalent:

(1) $H\left(|w|^{2}\right)+H\left(|\mathcal{F}(w)|^{2}\right)=-4\|w\|_{2}^{2} \log \|w\|_{2}$;

(2) $\mathcal{S}(w) \mathcal{S}(\mathcal{F}(w))=1$;

(3) $w$ is an extremal bi-partial isometry.

(4) $w$ is a bi-shift of a biprojection.

Conditions (1) and (2) are inequalities in general, namely Hirschman-Beckner uncertainty principle and Donoho-Stark uncertainty principle. When $\mathbb{G}$ has biprojections, the above four conditions characterize the minimizers of the Hirschman-Beckner uncertainty principle. In terms of these minimizers, we obtain Hardy's uncertainty principle for unimodular Kac algebras.

Main Theorem 2 (Hardy's uncertainty principle, Theorem 3.17). Let $\mathbb{G}$ be a unimodular Kac algebra. Suppose that a non-zero $w$ in $L^{1}(\mathbb{G}) \cap L^{\infty}(\mathbb{G})$ satisfies the conditions in Theorem 1 , For any $x \in L^{1}(\mathbb{G}) \cap L^{\infty}(\mathbb{G})$, if $|x| \leq C|w|$ and $|\mathcal{F}(x)| \leq C^{\prime}|\mathcal{F}(w)|$, for some constants $C>0$ and $C^{\prime}>0$, then $x$ is a scalar multiple of $w$.

Acknowledgements. Parts of the work was done during visits of authors to Hebei Normal University. The authors would like to thank Quanhua Xu for helpful discussions. Zhengwei Liu was supported by a grant from Templeton Religion Trust. Jinsong Wu was supported by NSFC (Grant no. A010602).

\footnotetext{
${ }^{1}$ Bisch and Jones introduced biprojections 3, 4] which generalize the indicator function of subgroups. Bi-shifts of biprojections generalize the notion of modulation and translation of the indicator function of subgroups, although modulation and translation do not make sense in subfactor theory.
} 


\section{Preliminaries}

Let $\mathcal{M}$ be a von Neumann algebra acting on a Hilbert space $\mathcal{H}$ with a normal semifinite faithful tracial weight $\varphi$.

A closed densely defined operator $x$ affiliated with $\mathcal{M}$ is called $\varphi$-measurable if for all $\epsilon>0$ there exists a projection $p \in \mathcal{M}$ such that $p \mathcal{H} \subset \mathcal{D}(x)$, and $\varphi(1-p) \leq \epsilon$, where $\mathcal{D}(x)$ is the domain of $x$. Denote by $\widetilde{\mathcal{M}}$ the set of $\varphi$-measurable closed densely defined operators. Then $\widetilde{\mathcal{M}}$ is $*$-algebra with respect to strong sum, strong product, and adjoint operation. If $x$ is a positive self-adjoint $\varphi$-measurable operator, then $x^{\alpha} \log x$ is $\varphi$-measurable for any $\alpha \in \mathbb{C}$ with $\Re \alpha>0$, where $\Re \alpha$ is the real part of $\alpha$.

The sets

$$
N\left(\varepsilon, \varepsilon^{\prime}\right)=\left\{x \in \widetilde{\mathcal{M}} \mid \exists \text { a projection } p \in \mathcal{M}: p \mathcal{H} \subseteq \mathcal{D}(x),\|x p\| \leq \varepsilon, \varphi(1-p) \leq \varepsilon^{\prime}\right\},
$$

where $\epsilon, \epsilon^{\prime}>0$, form a basis for the neighborhoods of 0 for a topology on $\widetilde{\mathcal{M}}$ that turns $\widetilde{\mathcal{M}}$ into a topological vector space. Now $\widetilde{\mathcal{M}}$ is a complete Hausdorff topological *-algebra and $\mathcal{M}$ is a dense subset of $\widetilde{\mathcal{M}}$.

For any positive self-adjoint operator $x$ affiliated with $\mathcal{M}$, we put

$$
\varphi(x)=\sup _{n \in \mathbb{N}} \varphi\left(\int_{0}^{n} t d e_{t}\right),
$$

where $x=\int_{0}^{\infty} t d e_{t}$ is the spectral decomposition of $x$. Then for $p \in[1, \infty)$, the noncommutative $L^{p}$ space $L^{p}(\mathcal{M})$ with respect to $\varphi$ is given by

$$
L^{p}(\mathcal{M})=\left\{x \text { densely defined, closed, affiliated with } \mathcal{M} \mid \varphi\left(|x|^{p}\right)<\infty\right\} .
$$

The $p$-norm $\|x\|_{p}$ of $x$ in $L^{p}(\mathcal{M})$ is given by $\|x\|_{p}=\varphi\left(|x|^{p}\right)^{1 / p}$. We have that $L^{p}(\mathcal{M}) \subseteq \widetilde{\mathcal{M}}$. For more details on noncommutative $\mathrm{L}^{p}$ space we refer to [26, 25].

Throughout the paper, we will use the results in [18] frequently. Let us recall the definition of locally compact quantum groups.

Let $\mathcal{M}$ be a von Neumann algebra with a normal semifinite faithful weight $\varphi$. Then $\mathfrak{N}_{\varphi}=\{x \in$ $\left.\mathcal{M} \mid \varphi\left(x^{*} x\right)<\infty\right\}, \mathfrak{M}_{\varphi}=\mathfrak{N}_{\varphi}^{*} \mathfrak{N}_{\varphi}, \mathfrak{M}_{\varphi}^{+}=\left\{x \geq 0 \mid x \in \mathfrak{M}_{\varphi}\right\}$. Denote by $\mathcal{H}_{\varphi}$ the Hilbert space by taking the closure of $\mathfrak{N}_{\varphi}$. The map $\Lambda_{\varphi}: \mathfrak{N}_{\varphi} \mapsto \mathcal{H}_{\varphi}$ is the inclusion map. We may use $\Lambda$ instead of $\Lambda_{\varphi}$ if there is no confusion.

A locally compact quantum group $\mathbb{G}=(\mathcal{M}, \Delta, \varphi, \psi)$ consists of

(1) a von Neumann algebra $\mathcal{M}$,

(2) a normal, unital, ${ }^{*}$-homomorphism $\Delta: \mathcal{M} \rightarrow \mathcal{M} \bar{\otimes} \mathcal{M}$ such that $(\Delta \otimes \iota) \circ \Delta=(\iota \otimes \Delta) \circ \Delta$,

(3) a normal, semi-finite, faithful weight $\varphi$ such that $(\iota \otimes \varphi) \Delta(x)=\varphi(x) 1, \forall x \in \mathfrak{M}_{\varphi}^{+}$;

a normal, semi-finite, faithful weight $\psi$ such that $(\psi \otimes \iota) \Delta(x)=\psi(x) 1, \forall x \in \mathfrak{M}_{\psi}^{+}$,

where $\bar{\otimes}$ denotes the von Neumann algebra tensor product, $\iota$ denotes the identity map. The normal, unital, *-homomorphism $\Delta$ is a comultiplication of $\mathcal{M}, \varphi$ is the left Haar weight, and $\psi$ is the right Haar weight. 
We assume that $\mathcal{M}$ acts on $\mathcal{H}_{\varphi}$. There exists a unique unitary operator $W \in \mathcal{B}\left(\mathcal{H}_{\varphi} \otimes \mathcal{H}_{\varphi}\right)$ which is known as the multiplicative unitary defined by

$$
W^{*}\left(\Lambda_{\varphi}(a) \otimes \Lambda_{\varphi}(b)\right)=\left(\Lambda_{\varphi} \otimes \Lambda_{\varphi}\right)(\Delta(b)(a \otimes 1)), \quad a, b \in \mathfrak{N}_{\varphi} .
$$

Moreover for any $x \in \mathcal{M}, \Delta(x)=W^{*}(1 \otimes x) W$.

For the locally compact quantum group $\mathbb{G}$, there exist an antipode $S$, a scaling automorphism group $\tau$ and a unitary antipode $R$ and there also exists a dual locally compact quantum group $\hat{\mathbb{G}}=(\hat{\mathcal{M}}, \hat{\Delta}, \hat{\varphi}, \hat{\psi})$ of $\mathbb{G}$. The antipode, the scaling group, and the unitary antipode of $\hat{\mathbb{G}}$ will denoted by $\hat{S}, \hat{\tau}$, and $\hat{R}$ respectively. We refer 18,19 for more details.

For any $\omega \in \mathcal{M}_{*}, \lambda(\omega)=(\omega \otimes \iota)(W)$ is the Fourier representation of $\omega$, where $\mathcal{M}_{*}$ is the Banach space of all bounded normal functional on $\mathcal{M}$. For any $\omega, \theta$ in $\mathcal{M}_{*}$, the convolution $\omega * \theta$ is given by

$$
\omega * \theta=(\omega \otimes \theta) \Delta .
$$

In [20], S. Wang and the authors defined the convolution $x * y$ of $x \in L^{p}(\mathbb{G})$ and $L^{q}(\mathbb{G})$ for $1 \leq p, q \leq 2$. If the left Haar weights $\varphi, \hat{\varphi}$ of $\mathbb{G}$ and $\hat{\mathbb{G}}$ respectively are tracial weights, we have that the convolution is well-defined for $1 \leq p, q \leq \infty$ by the results in [20].

For any locally compact quantum group $\mathbb{G}$, the Fourier transforms $\mathcal{F}_{p}: L^{p}(\mathbb{G}) \rightarrow L^{q}(\hat{\mathbb{G}})$ is welldefined. (See [6, [5] for the definition of Fourier transforms and [8] for the definition of the Fourier transform for algebraic quantum groups.) For any $x$ in $L^{1}(\mathbb{G})$, we deonte by $x \varphi$ the bounded linear functional on $L^{\infty}(\mathbb{G})$ given by $(x \varphi)(y)=\varphi(y x)$ for any $y$ in $L^{\infty}(\mathbb{G})$. Recall that a projection $p$ in $L^{1}(\mathbb{G}) \cap L^{\infty}(\mathbb{G})$ is a biprojection if $\mathcal{F}_{1}(p \varphi)$ is a multiple of a projection in $L^{\infty}(\hat{\mathbb{G}})$, (see [20] for more properties of biprojections).

\section{Main Results}

In this section, we will focus on a unimodular Kac algebra $\mathbb{G}$, which is a locally compact quantum group subject to the condition $\varphi=\psi$ is tracial. (See [13] for more details.) We denote $L^{\infty}(\mathbb{G})$ by $\mathcal{M}$. The Fourier transform $\mathcal{F}_{p}$ from $L^{p}(\mathbb{G})$ to $L^{q}(\hat{\mathbb{G}})$ is given by $x \mapsto \lambda(x \varphi)$ for any $x \in L^{1}(\mathbb{G}) \cap L^{\infty}(\mathbb{G})$. For a unimodular Kac algebra $\mathbb{G}$, we will denote by $\mathcal{F}$ the Fourier transform for simplicity.

For any $\varphi$-measurable element $x$ in $\widetilde{\mathcal{M}}$, the von Neumann entropy $H\left(|x|^{2}\right)$ is defined by

$$
H\left(|x|^{2}\right)=-\varphi\left(x^{*} x \log x^{*} x\right) .
$$

Proposition 3.1. Let $\mathbb{G}$ be a unimodular Kac algebra. Then for any $x \in L^{1}(\mathbb{G}) \cap L^{2}(\mathbb{G})$, we have

$$
H\left(|x|^{2}\right)+H\left(|\mathcal{F}(x)|^{2}\right) \geq-4\|x\|_{2}^{2} \log \|x\|_{2} .
$$

Proof. By Lemma 18 in [26], we have that $\alpha \mapsto|x|^{\alpha}$ is differentiable for $\alpha>0$. Now differentiating the Hausdorff-Young inequality [

$$
\|\mathcal{F}(x)\|_{q} \leq\|x\|_{p}, \quad x \in L^{1}(\mathbb{G}) \cap L^{2}(\mathbb{G}), \quad p \in[1,2], \quad \frac{1}{p}+\frac{1}{q}=1,
$$

with respect to $p$ and plug $p=2$ into the result inequality, we can obtain that

$$
H\left(|x|^{2}\right)+H\left(|\mathcal{F}(x)|^{2}\right) \geq-4\|x\|_{2}^{2} \log \|x\|_{2} .
$$


For any $x \in \widetilde{\mathcal{M}}$, let $\mathcal{S}(x)=\varphi(\mathcal{R}(x))$, where $\mathcal{R}(x)$ is the range projection of $x$.

Proposition 3.2. Let $\mathbb{G}$ be a unimodular Kac algebra. Then for any nonzero $x \in L^{1}(\mathbb{G}) \cap L^{2}(\mathbb{G})$, we have

$$
\mathcal{S}(x) \mathcal{S}(\mathcal{F}(x)) \geq 1
$$

Proof. We present two proofs here.

1. By using the inequality $\log \mathcal{S}(x) \geq H\left(|x|^{2}\right)$ when $\|x\|_{2}=1$ and Proposition 3.1 we see the proposition is true.

2. We assume that $\mathcal{S}(x), \mathcal{S}(\mathcal{F}(x))<\infty$. Then by Hölder's inequality, we have

$$
\begin{aligned}
\|\mathcal{F}(x)\|_{\infty} & \leq\|x\|_{1} \leq\|\mathcal{R}(x)\|_{2}\|x\|_{2} \\
& =\mathcal{S}(x)^{1 / 2}\|\mathcal{F}(x)\|_{2} \\
& \leq \mathcal{S}(x)^{1 / 2} \mathcal{S}(\mathcal{F}(x))^{1 / 2}\|\mathcal{F}(x)\|_{\infty} .
\end{aligned}
$$

Therefore $\mathcal{S}(x) \mathcal{S}(\mathcal{F}(x)) \geq 1$.

Definition 3.3. An element $x$ in $L^{1}(\mathbb{G}) \cap L^{2}(\mathbb{G})$ is said to be extremal if $\|\mathcal{F}(x)\|_{\infty}=\|x\|_{1}$. We say a nonzero element $x$ is an (extremal) bi-partial isometry if $x$ and $\mathcal{F}(x)$ are multiplies of (extremal) partial isometries.

Proposition 3.4. Let $\mathbb{G}$ be a unimodular Kac algebra. If $x$ is extremal, then $x^{*}$ and $R(x)$ are extremal.

Proof. By Proposition 2.4 in [19], we have

$$
\begin{aligned}
\left\|\mathcal{F}\left(x^{*}\right)\right\|_{\infty} & =\left\|\lambda\left(x^{*} \varphi\right)\right\|_{\infty}=\left\|\lambda\left(x^{*} \varphi\right)^{*}\right\|_{\infty} \\
& =\left\|\lambda\left(\overline{x^{*} \varphi} R\right)\right\|_{\infty}=\|\lambda(x \varphi R)\|_{\infty} \\
& =\|\hat{R}(\lambda(x \varphi))\|_{\infty}=\|\lambda(x \varphi)\|_{\infty}, \\
\|\mathcal{F}(R(x))\|_{\infty} & =\|\lambda(R(x) \varphi)\|_{\infty}=\|\lambda(x \varphi R)\|_{\infty} \\
& =\|\hat{R}(\lambda(x \varphi))\|_{\infty}=\|\lambda(x \varphi)\|_{\infty},
\end{aligned}
$$

and

$$
\varphi(|x|)=\varphi\left(\left|x^{*}\right|\right)=\varphi(R(|x|))=\varphi(|R(x)|)
$$

Therefore $x^{*}$ and $R(x)$ are extremal.

Proposition 3.5. Let $\mathbb{G}$ be a unimodular Kac algebra. For any nonzero $x$ in $L^{1}(\mathbb{G}) \cap L^{2}(\mathbb{G})$, the following statements are equivalent:

(1) $H\left(|x|^{2}\right)+H\left(|\mathcal{F}(x)|^{2}\right)=-4\|x\|_{2}^{2} \log \|x\|_{2}$;

(2) $\mathcal{S}(x) \mathcal{S}(\mathcal{F}(x))=1$;

(3) $x$ is an extremal bi-partial isometry.

Proof. " $(1) \Rightarrow(3) "$. We assume that $\|x\|_{2}=1$. Now we follow the proof in [17. First, we define a complex function $F(z)$ for $z=\sigma+i t, \frac{1}{2}<\sigma<1$ as

$$
F(z)=\hat{\varphi}\left(\mathcal{F}\left(w_{x}|x|^{2 z}\right)|\mathcal{F}(x)|^{2 z} w_{\mathcal{F}(x)}^{*}\right),
$$


where $w_{x}$ means the partial isometry in the polar decomposition of $x$. Note that $x \in L^{1}(\mathbb{G}) \cap L^{2}(\mathbb{G})$, we see that $\mathcal{F}\left(w_{x}|x|^{2 z}\right)$ is well-defined.

By Hölder's inequality and the Hausdorff-Young inequality [], we have

$$
|F(\sigma+i t)| \leq\left\|\mathcal{F}\left(w_{x}|x|^{2 z}\right)\right\|_{\frac{1}{1-\sigma}}\left\||\mathcal{F}(x)|^{2 z}\right\|_{\frac{1}{\sigma}} \leq\left\||x|^{2 \sigma}\right\|_{\frac{1}{\sigma}}\left\||\mathcal{F}(x)|^{2 \sigma}\right\|_{\frac{1}{\sigma}}=1 .
$$

This implies $F(z)$ is bounded on $\frac{1}{2}<\sigma<1$. By Lemma 18 in [26] again, we can follow the proof of Theorem 6.4 in [17] directly to obtain that

$$
\hat{\varphi}\left(\mathcal{F}(x|x|)|\mathcal{F}(x)| \mathcal{F}(x)^{*}\right)=1 .
$$

Now we see that

$$
\begin{aligned}
1 & =\hat{\varphi}\left(\mathcal{F}(x|x|)|\mathcal{F}(x)| \mathcal{F}(x)^{*}\right) \\
& =\left(x|x| \varphi \otimes\left(|\mathcal{F}(x)| \mathcal{F}(x)^{*}\right) \hat{\varphi}\right)(W) \\
& =\left(w_{x}|x|^{2} \varphi \otimes\left(|\mathcal{F}(x)|^{2} w_{\mathcal{F}(x)}^{*}\right) \hat{\varphi}\right)(W) \\
& =\left(|x|^{2} \varphi \otimes\left(|\mathcal{F}(x)|^{2}\right) \hat{\varphi}\right)\left(\left(1 \otimes w_{\mathcal{F}(x)}^{*}\right) W\left(w_{x} \otimes 1\right)\right) \\
& \leq\left(|x|^{2} \varphi \otimes\left(|\mathcal{F}(x)|^{2}\right) \hat{\varphi}\right)(1 \otimes 1)=1 .
\end{aligned}
$$

Let $p=w_{x}^{*} w_{x}$ and $q=w_{\mathcal{F}(x)}^{*} w_{\mathcal{F}(x)}$. Since the equality holds in Inequality (1), we have that

$$
\left(p \otimes w_{\mathcal{F}(x)}^{*}\right) W\left(w_{x} \otimes q\right)=p \otimes q .
$$

Applying $|x| \varphi \otimes \iota$ to the both sides of the equation above, we obtain that

$$
w_{\mathcal{F}(x)}^{*} \mathcal{F}(x) q=\varphi(|x|) q,
$$

i.e. $\mathcal{F}(x)=\varphi(|x|) w_{\mathcal{F}(x)}$. Similarly, we can obtain that $x=\hat{\varphi}(|\mathcal{F}(x)|) w_{x}$. Now we see that $x$ is an extremal bi-partial isometry.

$"(3) \Rightarrow(2) "$. Suppose $x$ is an extremal bi-partial isometry. Following the second proof in Proposition 3.2, we have

$$
\begin{aligned}
\|\mathcal{F}(x)\|_{\infty} & =\|x\|_{1}=\|\mathcal{R}(x)\|_{2}\|x\|_{2} \\
& =\varphi(\mathcal{R}(x))^{1 / 2}\|\mathcal{F}(x)\|_{2} \\
& =\varphi(\mathcal{R}(x))^{1 / 2} \hat{\varphi}(\mathcal{R}(\mathcal{F}(x)))^{1 / 2}\|\mathcal{F}(x)\|_{\infty} .
\end{aligned}
$$

Hence $\mathcal{S}(x) \mathcal{S}(\mathcal{F}(x))=1$.

$"(2) \Rightarrow(1) "$. Since (2) is weaker than (1), we see that (2) implies (1).

Definition 3.6. Let $\mathbb{G}$ be a unimodular Kac algebra with a biprojection $B$ in $L^{1}(\mathbb{G}) \cap L^{\infty}(\mathbb{G})$. A projection $x$ in $L^{1}(\mathbb{G}) \cap L^{2}(\mathbb{G})$ is called a left shift of a biprojection $B$ if $\varphi(x)=\varphi(B)$ and $x * B=\varphi(B) x$. A projection $x$ in $L^{1}(\mathbb{G}) \cap L^{2}(\mathbb{G})$ is called a right shift of a biprojection $B$ if $\varphi(x)=\varphi(B)$ and $B * x=\varphi(B) x$.

Proposition 3.7. Let $\mathbb{G}$ be a unimodular Kac algebra. Suppose that there exists a biprojection $B$ in $L^{1}(\mathbb{G}) \cap L^{\infty}(\mathbb{G})$ and $x$ is a right (or left) shift of a biprojection $B$ in $L^{1}(\mathbb{G}) \cap L^{2}(\mathbb{G})$. Then $x$ is an extremal bi-partial isometry. 
Proof. By Proposition 3.5 it suffices to show that $x$ is a minimizer of the uncertainty principle.

Since $B * x=\varphi(B) x$, we have $\mathcal{F}(B) \mathcal{F}(x)=\varphi(B) \mathcal{F}(x)$ i.e. $\mathcal{R}(\mathcal{F}(x)) \leq \mathcal{R}(\mathcal{F}(B))$.

By Proposition 3.2, we have $\varphi(x) \hat{\varphi}(\mathcal{R}(\mathcal{F}(x))) \geq 1$ and

$$
1=\varphi(B) \hat{\varphi}(\mathcal{R}(\mathcal{F}(B))) \geq \varphi(x) \hat{\varphi}(\mathcal{R}(\mathcal{F}(x))) \geq 1 .
$$

Now we have $\mathcal{R}(\mathcal{F}(x))=\mathcal{R}(\mathcal{F}(B))$. Hence $x$ is a minimizer of the uncertainty principle.

Definition 3.8. Let $\mathbb{G}$ be a unimodular Kac algebra. Suppose there exists a biprojection $B$ in $L^{1}(\mathbb{G}) \cap L^{2}(\mathbb{G})$, we denote by $\widetilde{B}$ the range projection of $\mathcal{F}(B)$. A nonzero element $x$ in $L^{\infty}(\mathbb{G})$ is said to be a bi-shift of a biprojection $B$ if there exist a right shift $B_{g}$ of the biprojection $B$ and a right shift $\widetilde{B}_{h}$ of the biprojection $\widetilde{B}$ and an element $y$ in $L^{\infty}(\mathbb{G})$ such that

$$
x=\widehat{\mathcal{F}}\left(\widetilde{B}_{h}\right) *\left(B_{g} y\right) .
$$

Now we will prove that the bi-shift of a biprojection described as above is a minimizer of the uncertainty principle. To see this, we need the following lemma.

Lemma 3.9. Let $\mathbb{G}$ be a unimodular Kac algebra. Suppose $x, y$ and $\mathcal{R}(x), \mathcal{R}(y)$ are in $L^{1}(\mathbb{G}) \cap$ $L^{\infty}(\mathbb{G})$. Then

$$
(x * y)(x * y)^{*} \leq\left\|\mathcal{R}\left(x^{*}\right)\right\|_{2}^{2}\left(x x^{*}\right) *\left(y y^{*}\right),
$$

and

$$
\mathcal{R}(x * y) \leq \mathcal{R}(\mathcal{R}(x) * \mathcal{R}(y)) .
$$

Proof. First, we assume that $x$ and $y$ are positive. Then $x \leq\|x\| \mathcal{R}(x)$ and $y \leq\|y\| \mathcal{R}(y)$. Now by computing the convolution [20, we obtain that

$$
\begin{aligned}
x * y & =((x \varphi) R \otimes \iota)(\Delta(y)) \\
& =\left(\left(x^{1 / 2} \varphi x^{1 / 2}\right) R \otimes \iota\right)(\Delta(y)) \\
& \leq\|y\|\left(\left(x^{1 / 2} \varphi x^{1 / 2}\right) R \otimes \iota\right)(\Delta(\mathcal{R}(y))) \\
& =\|y\| x * \mathcal{R}(y) \\
& =\|y\|(\iota \otimes \mathcal{R}(y) \varphi R)(\Delta(x)) \\
& \leq\|x\|\|y\| \mathcal{R}(x) * \mathcal{R}(y) .
\end{aligned}
$$

Therefore,

$$
\mathcal{R}(x * y) \leq \mathcal{R}(\mathcal{R}(x) * \mathcal{R}(y)) .
$$

When $x, y$ are in the general case, we will show that

$$
(x * y)(x * y)^{*} \leq\left\|\mathcal{R}\left(x^{*}\right)\right\|_{2}^{2}\left(x x^{*}\right) *\left(y y^{*}\right) .
$$

If this inequality (2) is true, then we can see that the second inequality in the Lemma is proved. By 
Lemma 9.5 in $\left[18\right.$ and $L^{1}(\mathbb{G}) \cap L^{\infty}(\mathbb{G}) \subset \mathfrak{N}_{\varphi}$, we have

$$
\begin{aligned}
R & \left(\left(x x^{*}\right) *\left(y y^{*}\right)\right) \\
& =R\left(\left(x x^{*} \varphi\right) R \otimes \iota\right)\left(\Delta\left(y y^{*}\right)\right) \\
& =\left(\iota \otimes \omega_{\Lambda(x), \Lambda(x)}\right)\left(\Delta\left(R(y)^{*} R(y)\right)\right. \\
& \geq \frac{1}{\left\|\mathcal{R}\left(x^{*}\right)\right\|_{2}^{2}}\left(\left(\iota \otimes \omega_{\Lambda(x), \Lambda\left(\mathcal{R}\left(x^{*}\right)\right)}\right) \Delta(R(y))\right)^{*}\left(\iota \otimes \omega_{\Lambda(x), \Lambda\left(\mathcal{R}\left(x^{*}\right)\right)}\right) \Delta(R(y)) \\
& =\frac{1}{\left\|\mathcal{R}\left(x^{*}\right)\right\|_{2}^{2}}(R(x * y))^{*} R(x * y) \\
& =\frac{1}{\left\|\mathcal{R}\left(x^{*}\right)\right\|_{2}^{2}} R\left((x * y)(x * y)^{*}\right),
\end{aligned}
$$

i.e.

$$
(x * y)(x * y)^{*} \leq\left\|\mathcal{R}\left(x^{*}\right)\right\|_{2}^{2}\left(x x^{*}\right) *\left(y y^{*}\right) .
$$

Proposition 3.10. Let $\mathbb{G}$ be a unimodular Kac algebra. Suppose $x$ is the bi-shift of the biprojection $B$ as in the Definition 3.8. Then $\mathcal{R}\left(x^{*}\right)=B_{g}$ and $\mathcal{R}(\mathcal{F}(x))=\widetilde{B}_{h}$. Moreover, $x$ is a minimizer of the uncertainty principles.

Proof. Note that $x=\widehat{\mathcal{F}}\left(\widetilde{B}_{h}\right) *\left(B_{g} y\right)$, we then have $\mathcal{F}(x)=\widetilde{B}_{h} \mathcal{F}\left(B_{g} y\right)$. This implies that $\mathcal{R}(\mathcal{F}(x)) \leq$ $\widetilde{B}_{h}$. From the fact that $\widetilde{B}_{h}$ is a right shift of the biprojection $\widetilde{B}$, we see $\hat{\varphi}\left(\widetilde{B}_{h}\right)=\hat{\varphi}(\widetilde{B})$.

On the other hand, we have $\mathcal{R}\left(\widehat{\mathcal{F}}\left(\widetilde{B}_{h}\right)\right)=\mathcal{R}(\widehat{\mathcal{F}}(\widetilde{B}))=\mathcal{R}(B)=B$ and by Lemma 3.9.

$$
\begin{aligned}
\mathcal{R}(x) & \left.\leq \mathcal{R}\left(\mathcal{R}\left(\widehat{\mathcal{F}}\left(\widetilde{B}_{h}\right)\right) * \mathcal{R}\left(B_{g} y\right)\right)\right) \\
& \leq \mathcal{R}\left(B * B_{g}\right)=B_{g}
\end{aligned}
$$

Now by Proposition 3.2, we see that

$$
\begin{aligned}
1 & \leq \varphi(\mathcal{R}(x)) \hat{\varphi}(\mathcal{R}(\mathcal{F}(x))) \leq \varphi\left(B_{g}\right) \hat{\varphi}(\widetilde{B}) \\
& =\varphi(B) \hat{\varphi}(\widetilde{B})=1
\end{aligned}
$$

Therefore all inequalities above must be equalities and $\mathcal{R}(x)=B_{g}$ and $\mathcal{R}(\mathcal{F}(x))=\widetilde{B}_{h}$. Moreover, $x$ is a minimizer of the uncertainty principles.

Proposition 3.11. Let $\mathbb{G}$ be a unimodular Kac algebra. Suppose $w$ is a partial isometry in $L^{1}(\mathbb{G}) \cap$ $L^{\infty}(\mathbb{G})$ and $\mathcal{F}(w)$ is extremal. Then $w$ is an extremal bi-partial isometry.

Proof. By Hölder's inequality, we have $x$ is a multiple of a partial isometry if and only if $\|x\|_{2}^{2}=$ $\|x\|_{\infty}\|x\|_{1}$. To see that $\mathcal{F}(w)$ is a multiple of a partial isometry, it is enough to check that

$$
\|\mathcal{F}(w)\|_{2}^{2}=\|\mathcal{F}(w)\|_{\infty}\|\mathcal{F}(w)\|_{1} .
$$

Since $\mathcal{F}(w)$ is extremal, we have

$$
\|w\|_{\infty}=\|\widehat{\mathcal{F}}(\mathcal{F}(w))\|_{\infty}=\|\mathcal{F}(w)\|_{1} .
$$


Now by Hölder's inequality and Hausdorff-Young inequality [6], we obtain

$$
\begin{aligned}
\|\mathcal{F}(w)\|_{\infty}\|\mathcal{F}(w)\|_{1} & \geq\|\mathcal{F}(w)\|_{2}^{2}=\|w\|_{2}^{2} \\
& =\|w\|_{\infty}\|w\|_{1} \\
& \geq\|\mathcal{F}(w)\|_{1}\|\mathcal{F}(w)\|_{\infty} .
\end{aligned}
$$

Hence $\|\mathcal{F}(w)\|_{2}^{2}=\|\mathcal{F}(w)\|_{\infty}\|\mathcal{F}(w)\|_{1}$ and $\|\mathcal{F}(w)\|_{\infty}=\|w\|_{1}$. Now we see that $w$ is an extremal bi-partial isometry.

Theorem 3.12. Let $\mathbb{G}$ be a unimodular Kac algebra. Suppose there is an extremal bi-partial isometry w in $L^{1}(\mathbb{G}) \cap L^{2}(\mathbb{G})$. Then

$$
\left(w * R(w)^{*}\right)\left(w^{*} * R(w)\right)=\|w\|_{2}^{2}\left(w w^{*}\right) *\left(R(w)^{*} R(w)\right) .
$$

Moreover $\frac{1}{\|w\|_{2}^{2}} w * R(w)^{*}$ is a partial isometry and $\|w\|_{1}=\frac{1}{\|w\|_{2}}\left\|w * R(w)^{*}\right\|_{1}$.

Proof. By Lemma 9.5 in [18, we have

$$
\begin{aligned}
R & \left(\left(w w^{*}\right) *\left(R(w)^{*} R(w)\right)\right) \\
& =R\left(\left(w w^{*} \varphi R \otimes \iota\right)\left(\Delta\left(R(w)^{*} R(w)\right)\right)\right) \\
& =\left(\iota \otimes\left(\omega_{\Lambda(w), \Lambda(w)}\right)\left(\Delta\left(w w^{*}\right)\right)\right) \\
& \geq \frac{1}{\|w\|_{2}^{2}}\left(\left(\iota \otimes \omega_{\Lambda(w), \Lambda(|w|)}\right) \Delta\left(w^{*}\right)\right)^{*}\left(\left(\iota \otimes \omega_{\Lambda(w), \Lambda(|w|)}\right) \Delta\left(w^{*}\right)\right) \\
& =\frac{1}{\|w\|_{2}^{2}}\left(R(w \varphi R \otimes \iota)\left(\Delta\left(R\left(w^{*}\right)\right)\right)\right)^{*}\left(R(w \varphi R \otimes \iota)\left(\Delta\left(R\left(w^{*}\right)\right)\right)\right) \\
& =\frac{1}{\|w\|_{2}^{2}} R\left(w * R\left(w^{*}\right)\right)^{*} R\left(w * R\left(w^{*}\right)\right) \\
& =\frac{1}{\|w\|_{2}^{2}} R\left(\left(w * R\left(w^{*}\right)\right)\left(w^{*} * R(w)\right)\right)
\end{aligned}
$$

i.e

$$
\left(w * R(w)^{*}\right)\left(w^{*} * R(w)\right) \leq\|w\|_{2}^{2}\left(w w^{*}\right) *\left(R(w)^{*} R(w)\right) .
$$

We will show that the traces of the both sides are equal. For the right hand side, we have

$$
\begin{aligned}
\varphi\left(\left(w w^{*}\right) *\left(R(w)^{*} R(w)\right)\right) & =\varphi\left(w w^{*}\right) \varphi\left(R(w)^{*} R(w)\right) \\
& =\|w\|_{2}^{2}\|R(w)\|_{2}^{2}=\|w\|_{2}^{4}
\end{aligned}
$$

On the other hand, since $w$ is an extremal bi-partial isometry, we let $w=\widehat{\mathcal{F}}(x)$ for $x$ in $L^{1}(\widehat{\mathbb{G}})$. Then we have that

$$
\mathcal{F}\left(w * R(w)^{*}\right)=\mathcal{F}(w) \mathcal{F}\left(R(w)^{*}\right)=x x^{*} .
$$

Therefore $w * R(w)^{*}=\widehat{\mathcal{F}}\left(x x^{*}\right)$ and

$$
\begin{aligned}
\varphi\left(\left(w * R(w)^{*}\right)\left(w^{*} * R(w)\right)\right) & =\varphi\left(\widehat{\mathcal{F}}\left(x x^{*}\right) \widehat{\mathcal{F}}\left(x x^{*}\right)^{*}\right) \\
& =\hat{\varphi}\left(x x^{*} x x^{*}\right) .
\end{aligned}
$$


Note that $x$ is a multiple of a partial isometry. We assume that $x=\mu x_{0}$ for some $\mu \in \mathbb{C}$ and a partial isometry $x_{0}$. Then $\left(x x^{*}\right)^{2}=|\mu|^{4}\left|x_{0}\right|$. Since $w$ is a minimizer of the uncertainty principle, we have $\varphi(|w|) \hat{\varphi}\left(\left|x_{0}\right|\right)=1$ i.e. $\hat{\varphi}\left(\left|x_{0}\right|\right)=\frac{1}{\|w\|_{2}^{2}}$. Meanwhile we have $\|w\|_{2}=\|x\|_{2}$. Now we can obtain that $\|w\|_{2}^{2}=|\mu|^{2} \frac{1}{\|w\|_{2}^{2}}$ and $|\mu|=\|w\|_{2}^{2}$.

Hence $\hat{\varphi}\left(\left(x x^{*}\right)^{2}\right)=|\mu|^{4} \frac{1}{\|w\|_{2}^{2}}=\|w\|_{2}^{6}$ i.e. the trace of the left hand side of inequality (3) is $\|w\|_{2}^{6}$. By Equation (4), we have the trace of the right hand side of inequality (3) is $\|w\|_{2}^{6}$. This implies that

$$
\left(w * R(w)^{*}\right)\left(w^{*} * R(w)\right)=\|w\|_{2}^{2}\left(w w^{*}\right) *\left(R(w)^{*} R(w)\right) .
$$

Now we show that $w * R(w)^{*}$ is a multiple of a partial isometry. By Hölder's inequality we have

$$
\|w\|_{2}^{6}=\left\|w * R(w)^{*}\right\|_{2}^{2} \leq\left\|w * R(w)^{*}\right\|_{\infty}\left\|w * R(w)^{*}\right\|_{1} .
$$

By Hausdorff-Young inequality [6], we obtain

$$
\left\|w * R(w)^{*}\right\|_{\infty}=\left\|\widehat{\mathcal{F}}\left(x x^{*}\right)\right\|_{\infty} \leq\left\|x x^{*}\right\|_{1}=\|x\|_{2}^{2}=\|w\|_{2}^{2}
$$

and by Young's inequality, we have

$$
\left\|w * R(w)^{*}\right\|_{1} \leq\|w\|_{1}\left\|R(w)^{*}\right\|_{1}=\|w\|_{1}^{2}=\|w\|_{2}^{4} .
$$

Hence all equalities of the inequalities above hold and

$$
\left\|w * R(w)^{*}\right\|_{2}^{2}=\left\|w * R(w)^{*}\right\|_{\infty}\left\|w * R(w)^{*}\right\|_{1} .
$$

Finally we see that $\frac{1}{\|w\|_{2}^{2}} w * R(w)^{*}$ is a partial isometry and

$$
\|w\|_{1}=\|w\|_{2}^{2}=\left\|\frac{1}{\|w\|_{2}^{2}} w * R(w)^{*}\right\|_{1}
$$

Corollary 6.12 in [17 is a useful tool to find an extremal bi-partial isometry in a given element. However, that result is not true in general. Instead, we have the following result for unimodular Kac algebras:

Corollary 3.13. Let $\mathbb{G}$ be a unimodular Kac algebra. Suppose $w \in L^{1}(\mathbb{G}) \cap L^{2}(\mathbb{G})$ such that $\left\|w * R\left(w^{*}\right)\right\|_{\infty}=\|w\|_{2}^{2},\|w\|_{2}^{2}$ is a point spectrum of $w * R\left(w^{*}\right)$, and $Q$ is the spectral projection of $\left|w * R\left(w^{*}\right)\right|$ with spectrum $\|w\|_{2}^{2}$. Then $Q$ is an extremal bi-partial isometry.

Proof. We assume that $\|w\|_{2}=1$. Note that

$$
\lim _{k \rightarrow \infty}\left(\left(w^{*} * R(w)\right)\left(w * R\left(w^{*}\right)\right)\right)^{k}=Q,
$$

in the strong operator topology and $Q$ is a projection. By the assumption that $w \in L^{1}(\mathbb{G}) \cap L^{2}(\mathbb{G})$ and Young's inequality, we have that $\left(\left(w^{*} * R(w)\right)\left(w * R\left(w^{*}\right)\right)\right)^{k} \in L^{1}(\mathbb{G})$ for $k=1,2, \ldots$ Hence $\lim _{k \rightarrow \infty}\left\|\left(\left(w^{*} * R(w)\right)\left(w * R\left(w^{*}\right)\right)\right)^{k}-Q\right\|_{1}=0$. By the Hausdorff-Young inequality [], we obtain that

$$
\lim _{k \rightarrow \infty}\left\|\mathcal{F}\left(\left(\left(w^{*} * R(w)\right)\left(w * R\left(w^{*}\right)\right)\right)^{k}\right)-\mathcal{F}(Q)\right\|_{\infty}=0
$$


i.e.

$$
\mathcal{F}(Q)=\lim _{k \rightarrow \infty}\left(\left(\mathcal{F}\left(w^{*}\right) \mathcal{F}\left(w^{*}\right)^{*}\right) *\left(\mathcal{F}(w) \mathcal{F}(w)^{*}\right)\right)^{*(k)}>0
$$

in the norm topology.

Note that $\left\|\left(\left(\mathcal{F}\left(w^{*}\right) \mathcal{F}\left(w^{*}\right)^{*}\right) *\left(\mathcal{F}(w) \mathcal{F}(w)^{*}\right)\right)^{*(k)}\right\|_{1}=\|w\|_{2}^{4 k}=1$. We then see that $\|\mathcal{F}(Q)\|_{1}=$ $1=\|Q\|_{\infty}$. By Proposition 3.11, we see that $Q$ is an extremal bi-partial isometry.

Theorem 3.14. Let $\mathbb{G}$ be a unimodular Kac algebra and $w \in L^{1}(\mathbb{G}) \cap L^{\infty}(\mathbb{G})$. Then $w$ is an extremal bi-partial isometry if and only if $w$ is a bi-shift of a biprojection. Furthermore, if $w$ is a projection, then it is a left (or right) shift of a biprojection.

Proof. Suppose $w$ is an extremal bi-partial isometry and $w$ is a partial isometry. Let

$$
B=\frac{1}{\|w\|_{2}^{4}}\left(w * R(w)^{*}\right)\left(w^{*} * R(w)\right) .
$$

By Theorem 3.12 we have that $\frac{1}{\|w\|_{2}^{2}} w * R(w)^{*}$ is a partial isometry and hence $B$ is a projection.

Now we compute the Fourier transform of $B$.

$$
\begin{aligned}
\mathcal{F}(B) & =\frac{1}{\|w\|_{2}^{4}} \mathcal{F}\left(\left(w * R(w)^{*}\right)\left(w^{*} * R(w)\right)\right) \\
& =\frac{1}{\|w\|_{2}^{2}} \mathcal{F}\left(\left(w w^{*}\right) *\left(R(w)^{*} R(w)\right)\right) \\
& =\frac{1}{\|w\|_{2}^{2}} \mathcal{F}\left(w w^{*}\right) \mathcal{F}\left(R(w)^{*} R(w)\right) \\
& =\frac{1}{\|w\|_{2}^{2}} \mathcal{F}\left(w w^{*}\right) \mathcal{F}\left(w w^{*}\right)^{*}
\end{aligned}
$$

Hence it is suffices to check $\mathcal{F}\left(w w^{*}\right)$ is a multiple of partial isometry. First we observe that $\mathcal{F}(w)$ is an extremal bi-partial isometry. By Theorem 3.12, we have that $\mathcal{F}(w) * \hat{R}\left(\mathcal{F}(w)^{*}\right)$ is a multiple of partial isometry and

$$
\mathcal{F}(w) * \hat{R}\left(\mathcal{F}(w)^{*}\right)=\mathcal{F}(w) * \mathcal{F}\left(w^{*}\right)=\mathcal{F}\left(w w^{*}\right) .
$$

Therefore $\mathcal{F}(B)$ is a multiple of a projection and $B$ is a biprojection.

Now we define $B_{g}=w w^{*}$, then $B_{g}$ is a projection. We are going to show that $B_{g}$ is a right shift of the biprojection $B$. By proposition 3.12 we have that $\frac{1}{\|w\|_{2}^{2}} B_{g} * R\left(B_{g}\right)=B$. Computing the trace on both sides, we have $\frac{1}{\|w\|_{2}^{2}} \varphi\left(B_{g}\right)^{2}=\varphi(B)$. Note that $\varphi\left(B_{g}\right)=\|w\|_{2}^{2}$, we see

$$
\varphi(B)=\frac{1}{\|w\|_{2}^{2}}\left(\|w\|_{2}^{2}\right)^{2}=\|w\|_{2}^{2}=\varphi\left(B_{g}\right) .
$$

Recall that $\mathcal{F}(w)$ is an extremal bi-partial isometry. We have $\|\mathcal{F}(w)\|_{\infty}=\|w\|_{1}$, and $\frac{1}{\|w\|_{2}^{2}} \mathcal{F}(w)$ is a partial isometry. By Theorem 3.12, we see that

$$
\frac{1}{\left\|\frac{1}{\|w\|_{2}^{2}} \mathcal{F}(w)\right\|_{2}^{2}} \frac{\mathcal{F}(w)}{\|w\|_{2}^{2}} * \frac{\hat{R}\left(\mathcal{F}(w)^{*}\right)}{\|w\|_{2}^{2}}=\frac{1}{\|w\|_{2}^{2}} \mathcal{F}\left(w w^{*}\right)=\frac{1}{\|w\|_{2}^{2}} \mathcal{F}\left(B_{g}\right)
$$

is a partial isometry. 
Hence we obtain that

$$
\begin{aligned}
\mathcal{F}\left(B_{g}\right) & =\frac{1}{\|w\|_{2}^{4}} \mathcal{F}\left(B_{g}\right) \mathcal{F}\left(B_{g}\right) * \mathcal{F}\left(B_{g}\right) \\
& =\frac{1}{\|w\|_{2}^{4}} \mathcal{F}\left(B_{g}\right) \mathcal{F}\left(R\left(B_{g}\right)\right) \mathcal{F}\left(B_{g}\right) \\
& =\frac{1}{\|w\|_{2}^{4}} \mathcal{F}\left(B_{g} * R\left(B_{g}\right) * B_{g}\right)
\end{aligned}
$$

and $\frac{1}{\|w\|_{2}^{4}} B_{g} * R\left(B_{g}\right) * B_{g}=B_{g}$. Then

$$
B * B_{g}=\frac{1}{\|w\|_{2}^{2}} B_{g} * R\left(B_{g}\right) * B_{g}=\|w\|_{2}^{2} B_{g}=\varphi\left(B_{g}\right) B_{g} .
$$

Therefore $B_{g}$ is a right shift of the biprojection $B$.

Let $\widetilde{B}_{h}=\frac{1}{\|w\|_{2}^{4}} \mathcal{F}(w) \mathcal{F}(w)^{*}$. We have $\widehat{\mathcal{F}}\left(\widetilde{B}_{h}\right)=\frac{1}{\|w\|_{2}^{4}} w * R(w)^{*}$. Finally we will find a form of $w$ in terms of $B_{g}$ and $\widetilde{B}_{h}$.

$$
\begin{aligned}
\mathcal{F}(w) & =\frac{1}{\|w\|_{2}^{4}} \mathcal{F}(w) \mathcal{F}(w)^{*} \mathcal{F}(w) \\
& =\frac{1}{\|w\|_{2}^{4}} \mathcal{F}(w) \mathcal{F}\left(R(w)^{*}\right) \mathcal{F}(w) \\
& =\frac{1}{\|w\|_{2}^{4}} \mathcal{F}\left(w * R(w)^{*} * w\right) .
\end{aligned}
$$

Then $w=\frac{1}{\|w\|_{2}^{4}} w * R(w)^{*} * w=\widehat{\mathcal{F}}\left(\widetilde{B}_{h}\right) *\left(B_{g} w\right)$.

Corollary 3.15. Let $\mathbb{G}$ be a unimodular Kac algebra. If $x \in L^{1}(\mathbb{G}) \cap L^{2}(\mathbb{G})$ and $\mathcal{F}(x)$ are positive and $\mathcal{S}(x) \mathcal{S}(\mathcal{F}(x))=1$, then $x$ is a biprojection.

Lemma 3.16. Let $\mathbb{G}$ be a unimodular Kac algebra. Suppose $B$ is a biprojection in $L^{1}(\mathbb{G}) \cap L^{\infty}(\mathbb{G})$ and $\widetilde{B}$ is the range projection of $\mathcal{F}(B)$ in $L^{1}(\widehat{\mathbb{G}}) \cap L^{\infty}(\widehat{\mathbb{G}})$. If $x \in L^{1}(\mathbb{G}) \cap L^{\infty}(\mathbb{G})$ such that $\mathcal{R}(x)=B$ and $\mathcal{R}(\mathcal{F}(x))=\widetilde{B}$, then $x$ is a multiple of $B$.

Proof. By the assumption, we have $B x=x$ and $\mathcal{F}(B) \mathcal{F}(x)=\varphi(B) \mathcal{F}(x)$, i.e. $B * x=\varphi(B) x$. Hence $B * B x=\varphi(B) x$. Note that $B$ is biprojection, then $B$ is a group-like projection [20] i.e.

$$
\Delta(B)(B \otimes 1)=\Delta(B)(1 \otimes B)=B \otimes B .
$$

Now we have

$$
\begin{aligned}
\varphi(B) x & =B *(B x)=(\varphi \otimes \iota)((B \otimes 1) \Delta(B x)) \\
& =(\varphi \otimes \iota)((1 \otimes B) \Delta(B) \Delta(x)) \\
& =\varphi(B x) B
\end{aligned}
$$

i.e. $x$ is a multiple of $B$. 
Theorem 3.17. [Hardy's uncertainty principle] Suppose $\mathbb{G}$ is a unimodular Kac algebra and $w \in \mathbb{G}$ is a bi-shift of biprojection. For any $x \in L^{1}(\mathbb{G}) \cap L^{\infty}(\mathbb{G})$, if $|x| \leq C|w|$ and $|\mathcal{F}(x)| \leq C^{\prime}|\mathcal{F}(w)|$, for some constants $C>0$ and $C^{\prime}>0$, then $x$ is a scalar multiple of $w$.

Proof. Suppose $w \in \mathbb{G}$ is a bi-shift of a biprojection $B$. Let $\widetilde{B}$ be the range projection of $\mathcal{F}(B)$, and $B_{g}, \widetilde{B}_{h}$ be right shifts of biprojections $B, \widetilde{B}$ respectively, such that $\mathcal{R}(w) \leq B_{g}$ and $\mathcal{R}(\mathcal{F}(w)) \leq \widetilde{B}_{h}$. If $x$ satisfies the assumption, then $\mathcal{R}(x) \leq B_{g}$ and $\mathcal{R}(\mathcal{F}(x)) \leq \widetilde{B}_{h}$. By Theorem $\mathbb{1}$ we have that $\mathcal{R}(w)=\mathcal{R}(x)=B_{g}$ and $\mathcal{R}(\mathcal{F}(w))=\mathcal{R}(\mathcal{F}(x))=\widetilde{B}_{h}$.

We assume that $x \neq 0$. Then $x w^{*}$ and $w w^{*}$ are nonzero and

$$
\begin{aligned}
\mathcal{R}\left(\mathcal{F}\left(x w^{*}\right)\right) & =\mathcal{R}\left(\mathcal{F}(x) * \mathcal{F}\left(w^{*}\right)\right) \\
& =\mathcal{R}\left(\mathcal{F}(x) * \hat{R}\left(\mathcal{F}(w)^{*}\right)\right. \\
& \leq \mathcal{R}\left(\widetilde{B}_{h} * \hat{R}\left(\widetilde{B}_{h}\right)\right) .
\end{aligned}
$$

By Theorem 3.12, $\widetilde{B}_{h} * \hat{R}\left(\widetilde{B}_{h}\right)$ is a multiple of a projection and

$$
\mathcal{S}\left(\mathcal{F}\left(x w^{*}\right)\right) \leq \mathcal{S}\left(\widetilde{B}_{h} * \hat{R}\left(\widetilde{B}_{h}\right)\right)=\mathcal{S}\left(\widetilde{B}_{h}\right)=\mathcal{S}(\mathcal{F}(w)) .
$$

Then

$$
1 \leq \mathcal{S}\left(x w^{*}\right) \mathcal{S}\left(\mathcal{F}\left(x w^{*}\right)\right)=\mathcal{S}\left(w x^{*}\right) \mathcal{S}\left(\mathcal{F}\left(x w^{*}\right)\right) \leq \mathcal{S}(w) \mathcal{S}(\mathcal{F}(w))=1
$$

Hence we have

$$
\mathcal{S}\left(w x^{*}\right)=\mathcal{S}(w) ; \quad \mathcal{S}\left(\mathcal{F}\left(x w^{*}\right)\right)=\mathcal{S}(\mathcal{F}(w))=\mathcal{S}\left(\widetilde{B}_{h} * \hat{R}\left(\widetilde{B}_{h}\right)\right)
$$

Therefore

$$
\mathcal{R}\left(w x^{*}\right)=\mathcal{R}(w)=\mathcal{R}(x)=\mathcal{R}\left(x w^{*}\right), \quad \mathcal{R}\left(\mathcal{F}\left(x w^{*}\right)\right)=\mathcal{R}\left(\widetilde{B}_{h} * \hat{R}\left(\widetilde{B}_{h}\right)\right) .
$$

Hence $x w^{*}$ is a bi-shift of a biprojection. Similarly $w w^{*}$ is a bi-shift of a biprojection. Moreover,

$$
\mathcal{R}\left(w x^{*}\right)=\mathcal{R}\left(w w^{*}\right), \quad \mathcal{R}\left(\mathcal{F}\left(x w^{*}\right)\right)=\mathcal{R}\left(\mathcal{F}\left(w w^{*}\right)\right) .
$$

By a similar argument, we have $\left(w x^{*}\right) * R\left(w w^{*}\right)^{*}$ and $\left(w w^{*}\right) * R\left(w w^{*}\right)^{*}$ are bi-shifts of biprojections and

$$
\begin{aligned}
\mathcal{R}\left(\left(x w^{*}\right) * R\left(w w^{*}\right)^{*}\right) & =\mathcal{R}\left(\left(w w^{*}\right) * R\left(w w^{*}\right)^{*}\right), \\
\mathcal{R}\left(\mathcal{F}\left(\left(x w^{*}\right) * R\left(w w^{*}\right)^{*}\right)\right) & =\mathcal{R}\left(\mathcal{F}\left(\left(w w^{*}\right) * R\left(w w^{*}\right)^{*}\right)\right) .
\end{aligned}
$$

By Theorem 3.12, we have that $\left(w w^{*}\right) * R\left(w w^{*}\right)^{*}$ is a multiple of a biprojection $Q$. By Lemma 3.16 and Equations (5), we have that $\left(x w^{*}\right) * R\left(w w^{*}\right)^{*}$ is a multiple of biprojection $Q$. Observe that both $x$ and $w$ are multiples of $\left(Q *\left(w w^{*}\right)\right) w$. Therefore $x$ is a scalar multiple of $w$.

Corollary 3.18. Let $\mathbb{G}$ be a unimodular Kac algebra. Suppose $B$ is a biprojection in $L^{1}(\mathbb{G})$ and $\widetilde{B}$ is the range projection of $\mathcal{F}(B)$ in $L^{1}(\widehat{\mathbb{G}})$. Let $B_{g}$ and $\widetilde{B}_{h}$ be right shifts of biprojections $B$ and $\widetilde{B}$ respectively. Then there is at most one element $x \in L^{1}(\mathbb{G}) \cap L^{2}(\mathbb{G})$ up to a scalar such that the range projection of $x$ is contained in $B_{g}$ and the range projection of $\mathcal{F}(x)$ is contained in $\widetilde{B}_{h}$.

Remark 3.19. Therefore we can use the supports $B_{g}$ and $\widetilde{B}_{h}$ to define a bi-shift of a biprojection. It is independent of the choice of $y$ in Definition 3.8 . 


\section{References}

[1] G. Alagic and A. Russell, Uncertainty principles for compact groups, Illinois J. Math. 52(2008), no. $4,1315-1324$.

[2] W. Beckner, Inequality in Fourier Analysis, Ann. Math. 102(1975), no.1, 159-182.

[3] D. Bisch, A note on intermediate subfactors, Pacific J. Math. 163(1994), 201-216.

[4] D. Bisch and V. Jones, Algebras associated to intermediate subfactors, Invent. Math. 128(1997), 89-157.

[5] M. Caspers, The $L^{p}$-Fourier transform on locally compact quantum groups, Journal of Operator Theory, 69(2013), 161-193.

[6] T. Cooney, A Hausdorff-Young inequality for locally compact quantum groups, International Journal of Mathematics, 21(2012), no. 12, 1619-1632.

[7] J. Crann and M. Kalantar, An uncertainty principle for unimodular locally compact quantum groups, J. Math. Phys. 55(2014), 081704.

[8] A. Van Daele, The Fourier transform in quantum group theory, ArXiv:math /0609502v3, 2007.

[9] D.L. Donoho and P.B. Stark, Uncertainty principles and signal recovery, SIAM J. Appl. Math. 49(1989), 906-931.

[10] M. Enock and J.-M. Schwartz, Une dulite dans les algebres de von Neumann, Note C.R. Acad. Sc. Paris, 277(1973), 683-685.

[11] M. Enock and J.-M. Schwartz, Une categorie d'algebres de Kac, Note C.R. Acad. Sc. Paris, 279(1974), 643-645.

[12] M. Enock and J.-M. Schwartz, Une dulite dans les algebres de von Neumann, Supp. Bull. Soc. Math. France Memoire, 44(1975), 643-645.

[13] M. Enock and J.-M. Schwartz, Kac algrbras and duality of locally compact groups, SpringerVerlag, 1992.

[14] M. Enock and R. Nest, Irreducible inclusions of factors, multiplicative unitaries and Kac algebras, Journal of Func. Anal. 137(1996), 466-543.

[15] G.H. Hardy, A theorem concerning Fourier transforms, Journal of the London Mathematical Society, 8(1933), no. 3, 227-231.

[16] I.I. Hirschman, A note on entropy, Amer. J. Math. 79(1957), 152-156.

[17] C. Jiang, Z. Liu and J. Wu, Noncommutative uncertainty principles, Journal of Functional Analysis, 270(2016), 264-311.

[18] J. Kustermans and S. Vaes, Locally compact quantum groups, Annales Scientifiques de l'Ecole Normale Superieure, 33(2000), no.6, 837-934. 
[19] J. Kustermans and S. Vaes, Locally quantum groups in the von Neumann algebraic setting, Math. Scand. 92(2003), no. 1, 68-92.

[20] Z. Liu, S. Wang and J. Wu, Young's inequality for locally compact quantum groups, preprint, 2015.

[21] M. Özaydm and T. Przebinda, An entropy-based uncertainty principle for a locally compact abelian group, J. Funct. Anal. 215(2004), 241-252.

[22] W. Szymanski, Finite index subfactors and Hopf algebra crossed products, Proc. Amer. Math. Soc. 120(1994), 519-528.

[23] K.T. Smith, The uncertainty principle on groups, SIAM J. Appl. Math. 50(1990), 876-882.

[24] T. Tao An uncertainty principle for cyclic groups of prime order, Math. Res. Lett. 12(2005), 121-127.

[25] M. Terp, Interpolation spaces between a von Neumann algebra and its predual, J. Operator Theory, 8(1982), 327-360.

[26] M. Terp, $L^{p}$ spaces associated von Neumann algebras, Preprint, 1981.

[27] L.I. Vainerman, Characterization of objects dual to locally compact groups, Funct. Anal. Appl., 8(1974), 66-67.

[28] L.I. Vainerman and G.I. Kac, Nonunimodular ring-groups and Hopf-von Neumann algebras, Soviet Math. Dokl. 23(1974), 1144-1148.

[29] L.I. Vainerman and G.I. Kac, Nonunimodular ring-groups and Hopf-von Neumann algebras, Math. USSR Sbornik, 23(1974), 185-214.

[30] W.H. Young, On the multiplication of successions of Fourier constants, Proc. Roy. Soc. London, Ser. A, 87(1912), 331-339. 